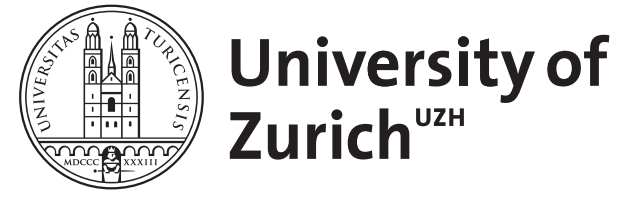

Zurich Open Repository and Archive

University of Zurich

University Library

Strickhofstrasse 39

CH-8057 Zurich

www.zora.uzh.ch

Year: 2019

\title{
Dinge als Aktanten in der Literatur
}

Messerli, Alfred

Posted at the Zurich Open Repository and Archive, University of Zurich

ZORA URL: https://doi.org/10.5167/uzh-184509

Journal Article

Published Version

Originally published at:

Messerli, Alfred (2019). Dinge als Aktanten in der Literatur. Kultur Korea:511-540. 


\section{Dinge als Aktanten in der Literatur}

Alfred Messerli (Uni Zürich, Schweiz)

)Wenn der Mensch von den Umständen gebildet wird, so muß man die Umstände menschlich bilden.«1) (Friedrich Engels, Karl Marx)

Mit der Zunahme der Gebrauchsgegenstände im 19. Jahrhundert in der westlichen Welt wurde die Abhängigkeit der Menschen von ihnen immer deutlicher. Zwar wissen wir die Physik, die Technik und die Sprache auf unserer Seite, die Dinge zu Gebrauchsdingen machen, oder sie zum unnützen Abfall erklären, oder ihnen, als Naturdinge, eine Existenzberechtigung zugestehen, sofern sie uns nicht stören oder zu unserem ästhetischen Gefallen beitragen.2) Die Dinge sind zu Sklaven der Menschen geworden. Dass sie sich dagegen auflehnen könnten, ist eine Katastrophenphantasie, die die Menschen seitdem nicht mehr loslässt und die Erhart Kästner in ")Aufstand der Dinge« (1973) literarisch gestaltete. Gerade die Vorstellung aber, dass die Dinge sich uns verweigern könnten, macht wiederum unsere Abhängigkeit von ihnen deutlich. In einem Vortrag vor den Studierenden der ETH-Zürich forderte Adolf Muschg 1983 einen anderen, einen neuen Umgang mit den Dingen. Den Anfang macht er mit dem Ding, das nicht funktioniert: »Das

1) Engels, Friedrich; Marx, Karl: Die heilige Familie, oder Kritik der kritischen Kritik. Gegen Bruno Bauer \& Consorten. Frankfurt am Main: Literarische Anstalt, 1845, 207.

2) Böhme, Hartmut: Fetischismus und Kultur. Eine andere Theorie der Moderne. Reinbek bei Hamburg: Rowohlt Taschenbuch Verl., (2006) ${ }^{2} 2006$ (Rowohlts Enzyklopädie; 55677), 42-43. 
verflixte Ding macht sich lustig über den Zweck, den wir ihm zugedacht haben, und gewinnt zugleich, wenn es uns Streiche spielt, einen Augenblick die Würde des Zwecklosen: es spielt mit uns. Dieser Augenblick der Beschämung für den Benützer könnte einer der Besinnung sein, denn das Objekt will sich offenbar nicht mar benützen lassen. Es spottet des gedankenlosen Anspruchs, dem wir es unterwerfen, mit dem wir Gebrauchsgegenstände behandeln: dass sie nichts für sich sein sollen, nur etwas für uns. Zugleich dämmert die Erinnerung an Zeiten auf, da die Sachen in der Tat als lebendige Begleiter unseres Alltags verehrt werden wollten, da wir sie darum bitten mussten, dass sie uns nicht auf den Kopf fielen. Da ist die Tücke des Objekts dann eine Mahnung an seinen magischen Ursprung, an die Tatsache, dass die Dinge, die uns umgeben, weil wir uns mit ihnen umgeben haben, leben.(13) In diesem Plädoyer an die Ingenieure, dass wir die Dinge formen und diese wiederum uns, wird unser Regime über sie in Frage gestellt. Über diesen Zusammenhang hat bereits in den 1920er Jahren ein Aleksander Gabricesvkij, der der russischen Avantgarde zuzurechnen ist, nachgedacht. Wir bemerkten an einem Ding, schreibt er, »nur jene Merkmale oder jene begrenzte Gruppe von Merkmalen«, die es uns erlaubten, in ihm »das bekannte und erprobte Werkzeug anzuerkennen«. Und weiter: »Wenn wir es benützen, schweigt das Ding.« Er rät uns deshalb, das Ding kontemplativ zu betrachten und seinen Nutzen zu vergessen und »die Vorstellung seiner Zweckmäßigkeit火. Und indem wir es in eine »bestimmte, handlungsabstinente, inaktuelle Sphäre« überführten, begimne das Ding »mit tausend Stimmen zu sprechen.« Es erzähle uns »von seinem Platz und von seiner Welt, von seinem Verhältnis zu uns und $\mathrm{zl}$ anderen Dingen, von seiner Geschichte, von seiner kulturellen

3) Muschg, Adolf: Die Tücke des verbesserten Objekts. Wald: Verl. im Waldgut, 1980 (Die Reihe im Waldgut; 3), 5-6.
Vergangenheit, Gegenwart und Zukunft; ohne von uns eine direkte physische Handlung $\mathrm{zu}$ verlangen, erscheint es zugleich eingebunden in das überaus reiche System von Beziehungen zu uns und zur übrigen Welt«.4) Die Unterscheidung in "Zuhandenheit« und »Vorhandenheit«, die Martin Heidegger in Sein und Zeit (1927) vornimmt, geht in eine ähnliche Richtung: Im Modus der »Zuhandenheit« dient das ,Zeug' (Artefakt) unauffällig und unscheinbar; erst wenn eine Störung aufritt, es fehlt, defekt ist, kommt seine Dinglichkeit zum Vorschein, seine bloße »Vorhandenheit(«.5)

Entdeckt wurden also der »Eigensinn der Dinge«(6) und der Umstand, dass Dinge vielseitig sind, aber selektiv wahrgenommen werden. Hier kommt nun die Literatur ins Spiel. Weil den Dingen in Texten das Entscheidende, sihre Dinglichkeit, ihre Materialität» fehlt, da »Dinge in Texten [...] keinem menschlichen Sinn unmittelbar zugänglich« sind, wird die Repräsentation von Dingen, ihre »Sinnlichkeit, Unmittelbarkeit und Materialität [...] zu einem zentralen Motiv der literarischen Dingrepräsentation. «7) Gerade deshalb spielt für Dorothee Kimmich die Textwissenschaft im Zusammenhang mit dem material turn eine besondere Rolle.8) Sie ist das wichtige Medium, in

4) Gabricesvkij, Aleksander: Die Sprache der Dinge. In: Hennig, Anke (ed.): Über die Dinge. Texte der russischen Avantgarde. Hamburg: Philo Fine Arts, 2010 (Findus-Bücher; 181), 567-579, hier 575.

5) Steiner, Uwe C.: Die Verrückung der anthropologischen Matrix. Vom Verhalten der Dinge bei Franz Kafka. In: Brunner, José (ed.): Erzählte Dinge. Mensch-Objekt-Beziehungen in der deutschen Literatur. Göttingen: Wallstein, 2015 (Schriftenreihe des Minerva Instituts für deutsche Geschichte der Universität Tel Aviv; 32), 159-176, hier 170 .

6) Hahn, Hans Peter: Vom Eigensinn der Dinge. In: Bayerisches Jahrbuch für Volkskunde (2013), 13-22, hier 18: »Es geht einerseits darum, was Menschen mit den Dingen machen, andererseits aber auch darum, was Dinge mit den Menschen machen."

7) Kimmich, Dorothee: Dinge in Texten. In: Scholz, Susanne; Vedder, Ulrike (eds.): Handbuch Literatur und Materielle Kultur. Berlin/Boston: De Gruyter, 2018 (Handbücher zur kulturwissenschaftlichen Philologie; 6), 21-28, hier 21. 
welchem die »Probleme« mit den Dingen, ihr Eigensinn, ihre unbekannte Seite, ihre "Ambivalenz von Zeichenhaftigkeit und zugleich nicht Zeichenhaftigkeit«, ihre Opazität, ihre Geschichte reflektiert und auserzählt werden kann.9) »Zunehmend火, schreibt Raphaela Knipp, »entdeckt die Literaturwissenschaft Dinge als Akteure im Sinne [Bruno] Latours und die Literatur als ein genuines Wissensgebiet dieser Erkenntnis.«10)

Im Folgenden möchte ich an ausgewählten literarischen Texten »lebendige Dinge«, die die »Grundregeln moderner Kommunikation und Verständigung überhaupt« verletzten, untersuchen. Denn, so schreibt Dorothee Kimmich, "Grenzziehungen zwischen Lebendigem und Leblosem und die Verletzung dieser Grenze zugleich scheinen also gerade das Neuartige der Moderne zu charakterisieren. $(11)$

Aktive Gegenstände im Märchen: Märchen können wie »kaum eine Gattung Unmöglichkeiten, Beschränkungen und Diskrepanzen austarieren« und beruhen in ihrem Kern »auf einer asymmetrischen Anlage von Wunsch und Erfüllung«.12) Ich wähle das kurze Märchen »Herr Korbes« aus den Kinderund Hausmärchen von Jacob und Wilhelm Grimm, das von der 1. Auflage (1812) bis zur 7. Auflage (1857) die Nummer 41 ist. Wenn auch oft ein

8) Kimmich, Dorothee: Der Fremde und seine Dinge. Bemerkungen zur Funktion fremder Dinge in der Literatur der Moderne. In: Brunner 2015 (wie Anm. 5), 177188, hier 182.

9) Kimmich 2018 (wie Anm. 7), 21. Vgl. Vedder, Ulrike: Sprache der Dinge. In: Scholz/Vedder 2018 (wie Anm. 7), 29-37.

10) Knipp, Raphaela: Narrative der Dinge. Literarische Modellierungen von Mensch-Ding-Beziehungen. In: Zeitschrift für Literaturwissenschaft und Linguistik 42. Jg., H. 168 (2012), 46-61, hier 53.

11) Kimmich, Dorothee: Lebendige Dinge in der Moderne. Konstanz: Konstanz University Press, 2011, 13.

12) Körte, Mona: Volks- und Kunstmärchen. In: Scholz/Vedder 2018 (wie Anm. 7), 214-221, hier 218.
Ding im Märchen ein Katalysator, ein Träger und Motor der Handlung ist, das dem Helden dient, haben wir hier ganz andere Verhältmisse. Es beginnt zunächst ganz harmlos nach dem Muster einer Tierfabel, die als Gleichniserzählung sich sprechender Tiere, Pflanzen, Dinge oder gar Körperteile »bedient«, um etwas Anderes damit evident zu machen. Mit diesem Muster wird im Märchen »Herr Korbes« radikal gebrochen.13) Da wollen ein Hühnchen und ein Hähnchen zusammen eine Reise machen. Hähnchen baut einen Reisewagen, der von vier Mäusen gezogen wird. Auf der Reise begegnet sie einer Katze, einem Mühlstein, dann einem Ei, einer Ente, einer Stecknadel und zuletzt einer Nähnadel. Die bitten alle, mitgenommen zu werden, was ihnen erlaubt wird. Als Reiseziel wird das Haus von Herrn Korbes angegeben. Dort angelangt, ist Herr Korbes nicht da. Tiere und Dinge nehmen das Haus regelrecht in Besitz und legen sich nun an verschiedenen Orten zur Ruhe, die Katze ins Kamin, die Ente ins Wasserschiff in der Küche, das Ei wickelte sich in das Handtuch, die Stecknadel steckte sich ins Stuhlkissen, die Nähnadel sprang »mitten ins Kopfkissen« und der Mühlstein legte sich über die Haustür: »Da kam der Herr Korbes nach Haus, gieng ans Kamin und wollte Feuer anmachen, da warf ihm die Katze das Gesicht voll Asche. Er lief geschwind in die Küche und wollte sich abwaschen, da sprützte ihm die Ente Wasser ins Gesicht. Er wollte sich an dem Handtuch abtrocknen, aber das Ei rollte ihm entgegen, zerbrach und klebte ihm die Augen zu. Er wollte sich ruhen, und setzte sich auf den Stuhl, da stach ihn die Stecknadel. Er gerieth in Zorn, und warf sich aufs Bett, wie er aber den Kopf aufs Kissen niederlegte, stach ihn die Nähnadel, so daß er aufschrie und ganz wüthend in die weite Welt laufen wollte. Wie er aber an die Hausthür kam, sprang der Mühlstein herunter und

13) Körte, Mona: Der Un-Sinn der Dinge in Märchentexten um 1800. In: Zeitschrift für Germanistik, NF 22 (2012), 57-71, hier 65. 
schlug ihn todt. Der Herr Korbes muß ein recht böser Mann gewesen sein. «14) Hier also treten Dinge (und Haustiere) als störrisch, neckisch, rebellisch, ja mörderisch auf. Es handelt sich um Artefakte mit einer gleichsam forcierten Materialität (dünn und spitz, rund und schwer), die sich nicht mehr zu dem gebrauchen lassen wollen, wozu sie geschaffen wurden.15) »Hier gehen nicht Menschen mit Dingen, sondern dezidiert Dinge mit Menschen um.«16) Die Märchendinge haben hier keine Geschichte. Sie ragen ins Märchengeschehen hinein, »um ihre Rolle [...] als Teil eines Mordkomplotts [...] mit höchster Präzision zu erfuillen.«17) Die Hilflosigkeit der Grimms diesem Märchen gegenüber zeigt sich auch im Umstand, dass der letzte Satz ( $)$ Der Herr Korbes muß ein recht böser Mann gewesen sein«) erst ab der 6 . Auflage von 1850 , gleichsam als Rationalisierung und Rechtfertigung eines Märchens ,ohne Sinn', von Wilhelm Grimm hinzugefugt wurde.

Dingbiografien: Nach Michael Niehaus haben Dinge kein Leben. "Sie werden nicht geboren, und sie sterben nicht.« Mit der Vorstellung der Objektbiographie werde den Objekten ein Leben lediglich »angedichtet«.18) Solche Lebensläufe hat Hans Christian Andersen in seinen Kunstmärchen Alltagsgegenständen oder Spielzeug zugestanden. Als »Dingmärchen« wird jene Gruppe von Märchen bezeichnet, in denen Andersen häusliche Gebrauchsgegenstände von ihrem Zerfall und Ausschluss »aus der behüteten

14) Grimm, [Jacob und Wilhelm]: Kinder und Hausmärchen. Gesammelt durch die Brüder G. Große Ausgabe. 2. Bde. Göttingen: Verlag der Dieterichschen Buchhandlung, (1812/1815) ${ }^{7} 1857$, Bd. 1, 215.

15) Vgl. Körte 2012 (wie Anm. 13), 58. Körte, Mona: Dinge im Diminutiv. Der Eigensinn des Kleinen in der »Kinder- und Hausmärchen« der Brüder Grimm. In: Brunner 2015 (Anm. 5), 21-36, 25-27.

16) Körte 2015 (wie Anm. 15), 28

17) Körte 2012 (wie Anm. 13), 69

18) Niehaus, Michael: Objektbiografien. In: In: Scholz/Vedder 2018 (wie Anm. 7), 239247 , hier 239.
Welt des bürgerlichen Interieurs« erzählen lässt und »den Leser in die verborgenen Räume der Städte - den Rinnstein, die Kanalisation, den Müllhaufen, die Verbrennungsöfen oder Schmelztiegel« führt. ${ }^{19)}$ Das fehlende Glied, der Sprung im Porzellan, kurz: Not und Differenzerfahrung stimulieren die Selbstreflexion.20) Einmal aus der Ordnung des Alltags geworfen, werden die subtilen, mitunter etwas eingebildeten, Beobachter ihrer selbst leise von einem heterodiegetischen Erzähler korrigiert. So setzt ein heiratsfähiger "Halskragen« zunächst einem schlanken Strumpfhalter zu, flirtet mit einem heißen Plätteisen, charmiert eine Papierschere und schließlich einen Kamm, bis er am Ende im Sack des Papiermüllers landet, wo ihm nichts bleibt, als mit seinen vielen Liebschaften $\mathrm{zu}$ prahlen: "'Ich habe viel auf meinem Gewissen; es thut mir noth, daß ich weißes Papier werde!' Und dahin gelangte der Halskragen; alle die Lumpen wurden weißes Papier, aber der Halskragen wurde gerade das Stück Papier, das wir hier sehen, worauf diese Geschichte gedruckt worden ist. - Und dies geschah deswegen, weil er hinterher so schrecklich mit Dingen prahlte, die gar nicht wahr gewesen. $\left\langle 2^{21)}\right.$ Andersen Märchen endet mit der Mahnung an den Leser, es nicht wie der Halskragen zu machen, »denn wir können in der That gar nicht wissen, ob wir nicht auch einmal in den Lumpensack kommen und zu dem weißen Papier umgemacht werden, worauf man unsere Geschichte, selbst die allergeheimste, abdruckt, so dass wir ebenfalls umherlaufen und sie, wie der

19) Müller-Wille, Klaus: Collagen, Wortdinge und stumme Bücher. Hans Christian Andersens (inter)materielle Poetik: Strässle, Thomas; Kleinschmidt, Christoph; Mohs, Johanne (eds.): Das Zusammenspiel der Materialien in den Künsten. Theorien - Praktiken - Perspektiven. Bielefeld: transcript Verl., 2013, 183-219, hier 207.

20) Für das Folgende vgl. Körte 2018 (wie Anm. 12), 215-216.

21) Andersen, H.[ans] C.[hristian]: Der Halskragen [1847]. In: ders.: Ausgewähite Werke, Bd. 5: Gesammelte Märchen. Leipzig: Verl. Carl B. Lorck, 1849, 533-536, hier 536. 
Halskragen, erzählen müssen.«22)

In diesem Märchen von Andersen erhält ein Gegenstand eine Biographie. Nach Auskunft von Wikipedia ist "[e]ine Biografie (auch Biographie, griechisch Broxpaфia, von Bios, bíos Leben und -graphie von ypd фo, gráphō ritzen, malen, schreiben) [‥] die Lebensbeschreibung einer Person«. Indem dieses Konzept auf einen Gegenstand übertragen wird, hat nun auch eine Sache ein bíos, ein Leben. Nach Hans Peter Hahn, einem Ethnologen, ist die Beobachtung, »dass Dinge einen Lebenslauf haben, dass sie verschiedene lange Lebensspannen durchlaufen, und dass sie in diesem Zeitraum in ganz verschiedene Kontexten verwendet werden«, ein zentraler Aspekt in der Wahmehmung materieller Kultur.23) Die sich ablösende ,Lebensphase' des Halskragens, der in weißes Papier umgewandelt wird, rufen christliche Bilder der Sündhaftigkeit und der Vergebung auf. Das einzige, was irritiert, ist der Umstand, dass der Halskragen nicht für seine Taten, die er nicht begangen hat, sondern für die Prahlerei, sie gemacht zu haben, in weißes Papier umgewandelt (bestraft bzw. geläutert) und mit seiner Biografie (»unsere Geschichte, selbst die allergeheimste«) bedruckt wird.

Dank den Forschungen von Max Blackwell wissen wir, dass schon zwischen 1750 und 1850 in der englischen Literatur so genannte It-Narratives auftreten, in denen Dinge, Gegenstände, Objekte in der dritten Person Singular »ihre eigene Geschichte erzählen, von ihren erduldeten ,Abenteuern Zeugnis ablegen. (24) Dieses Genre tritt um 1800 auch in die Kinderliteratur auf. Heinrich Böll führt um die Mitte des 20. Jahrhunderts die Tradition der

22) Andersen 1849 (wie Anm. 21), 536

23) Hahn, Hans Peter: Materielle Kultur. Eine Einführung. Berlin: Reimer, 2005, 40.

24) Michael Niehaus: Dinge in Bewegung. In: Samida, Stefanie; Eggert, Manfred K H.; Hahn, Hans Peter (eds.): Handbuch Materielle Kultur. Bedeutungen, Konzepte, Disziplinen. StuttgartWeimar: Metzler Verl., 2014, 132-140, hier 139. Vgl. Blackwell, Mark; Bellamy, Liz; Keenleyside, Heather; Lupton, Christina (eds.): British it-narratives, 1750-1830, 4 Bde. London: Pickering \& Chatto, 2012.
It-Narratives weiter, so in der Erzählung »Abenteuer eines Brotbeutels« (1951) und "Schicksal einer henkellosen Tasse« (1952). Im Falle der lädierten Tasse erzählt diese selber in einer Art innerem Monolog ihre Geschichte (»Mein Vater war ein Kuchenteller und meine Mutter eine ehrbare Butterdose; $[. .$.$] k).25) Thre Geschichte ist die der wechselnden Besitzer$ bzw. Verwender und der wechselnden Verwendungszwecke: Sie wurde benutzt, um daraus Kaffee, Kakao, Milch, Wein und Schnaps zu trinken (was sie kränkte), sie diente einem Knaben als Futterkrippe für seine Haustiere, als Badewanne furr seine winzige Puppe, sie diente ihm zum Anrühren von Farben, von Kleister und als Behälter von Seifenlauge, um Seifenblasen zu machen, und dem Vater als Topf für Rasierseife. Auch der Schriftsteller Tim Krohn hat kürzlich die »Objektbiographie« einer Matratze geschrieben. ${ }^{26)}$ Festzuhalten ist: Die ,Helden“ von Objektbiographien sind ganz und gar passiv. Sie sind keine märchenhaften Wesen, die ihren Objektstatus überschreiten und sich selbst auf den Weg machen können, wie Strohhalm, Kohle und Bohne im gleichnamigen Märchen von Jacob und Wilhelm Grimm (KHM 18). ${ }^{27)}$ In eben diesem Sinne ist "Der Halskragen« von Andersen als Kunstmärchen eine Objektbiographie oder »Dinggeschichte« (Michael Niehaus), und notwendigerweise erleiden hier die Dinge ihr Schicksal passiv und gestalten es nicht aktiv.

Die Tücke des Objekts: Als Redensart ist »Die Tücke des Objekts« so

25) Böll, Heinrich: Schicksal einer henkellosen Tasse. In: ders.: Gesammelte Erzählungen, 2. Bde. Köln: Kiepenheuer \& Witsch, 1981, Bd. 1, 368-374, hier 368.

26) Krohn, Tim: Aus dem Leben einer Matratze bester Machart. Zürich: Diogenes Verl., (2014) 2015.

27) Niehaus, Michael: Objektbiografien. In: In: Scholz/Vedder 2018 (wie Anm. 7), 239 247, hier 242. Vgl. auch Niehaus, Michael: Das Buch der wandemden Dinge. Vom Ring des Polykrates bis zum entwendeten Brief. München: Carl Hanser Verl., $2009,32-33$. 
geläufig, dass ihr Sinn schon wieder verstellt ist, denn: »die Tücke des Objekts ist zunächst eines des Subjekts Vischer und seiner Fehlleistungen. Er verschnitzt seine Schreibfeder, kleckst, wirft das Tintenfass um, weil er nicht schreiben will. [...] Oder: er verlegt alles, weil er nicht abzureisen wünscht. So unbequem und schmerzlich die Folgen solcher grandiosen Verdrängungsarbeit sind, so bequem sind sie wiederum fürs Subjekt. Denn wenn es seine Mängel den Objekten als Schuld zuschiebt, braucht es sich selbst nie $z u$ verändern.( $(28)$ Weiblichen Ursprungs hat die Tücke es auf den Mann abgesehen, dessen Ideale von soldatischer Männlichkeit sie demontiert: Das Kreuz mit den Hemdknöpfen lässt ihn endlich für die Ehe schwach werden. Opfer sucht sich die Tücke des Objekts im Bürgertum und im Gelehrtenstand; das Proletariat hat keinerlei Probleme mit Hemdknöpfen und Schreibfedern, sund verfugt nicht über das Regulativ des Kapitals, das es ihm ermöglichen könnte, die verhassten Objekte, die Brillen, Uhren, Geschirr zu exekutieren.(129) In zahlreichen Briefen, vor allem aber in seinem Roman, Auch Einer (1878), hat Friedrich Theodor Vischer eine Phänomenologie und eine Theorie der Tücke des Objekts entwickelt. Danach sind die Objekte von übelwollenden Dämonen beseelt. Sie zetteln den Aufstand des munteren Stockwerks«, der Welt der Materie und der bedeutungslosen Kleinigkeiten, gegen das obere an, ygegen die hehren Zwecksetzungen in Staat, Recht, Religion, Kunst und Kultur«,30) Und doch weiß Einhart, der Held von Auch Einer, dass es sich um ein mythologisches Konstrukt handelt, denn dieses sei »nur sprach- und phantasiegemäßß zu verstehen. Indem er die eigenen Projektionen erkennt und die "poetische Verwechslung von Subjekt und

28) Vischer, Friedrich Theodor. Ed. Ulrich Ott. Marbach: Deutsche Schillergesellschaft Marbach am Neckar, 1987 (= Marbacher Magazin, 44), 59.

29) Vischer 1987 (wie Anm. 28), 59.

30) Steiner, Uwe C.: Die Tücke des Objekts. Friedrich Theodor Vischers Auch Einer (1878). In: Scholz/Vedder 2018 (wie Anm. 7), 249-256, hier 250.
Objekt« als Anthropomorphismus durchschaut, setzt er die überkommene Hierarchie wieder in ihr Recht und rettet so »die anthropische Denkform«.31) Ludwig Wittgenstein kritisierte diese Tücke des Objekts als »dummen Anthropomorphismus«, und selbst Vischer selber würde ihm zustimmen, »dass die eigenen Ungeschicklichkeiten den Dingen als ihre Ranküne vorzuwerfen eine Projektion ist.« Es sei indes, meint Hartmut Böhme, "witzlose Aufklärung«, einen solchen »ubiquitären Mechanismus als dumm zu bezeichnen, während Vischer aus ihm genau seinen witzigen Roman macht. «32)

Erfahrung der Fremdheit im Eigenen: Alfred Polgar beschreibt in seinem kurzen Prosatext, wie unheimlich die Welt der Dinge sein kann. Die Dinge sind bei Polgar keine »Dinge an sich» und auch keine »Dinge für uns«, sondern $»$ Dinge für sich«. »Wenn die Dinge ,für sich sind, sind sie nicht nur allein, sondern auch ,autonom'. Sie folgen einer Eigenlogik, die sie weit entfernt von der Erkenntnis- und Wahrnehmungsfähigkeit des Menschen火, wodurch sie fremd und gefährlich erscheinen. ${ }^{33)}$ Der Mensch ist nicht Herr im eigenen Haus, denn dort haben die Dinge die Herrschaft inne: »Ich liebe die Einsamkeit, aber die Einsamkeit meines Zimmers liebe ich nicht. Weil ich tiefes Mißtrauen gegen die Dinge in ihm, gegen Wände, Möbel, Bilder habe und mich ihnen ausgeliefert fühle. Es sind viele gegen einen. Ich spüre, daß sie mich anstarren und ahne Zeichen der Verständigung zwischen ihnen und pfeife sorglos, um ihnen zu zeigen, daß ich mich gar nicht fürchte. Niemals öffne ich nachts, heimkehrend, die Wohnungstür, ohne ein wenig absichtlichen Lärm zu machen. Ich will nicht überraschen, besser: ich will

31) Steiner 2018 (wie Anm. 30), 251.

32) Böhme 2006 (wie Anm. 2), 493.

33) Kimmich 2018, 26; vgl. auch Kimmich 2015, 187. 
nicht überrascht werden. Wurde meine Abwesenheit vielleicht genutzt, um Unfug zu treiben, so sollen sie, rechtzeitig von meiner Nähe unterrichtet, noch Zeit haben, wieder in ihre gewohnte dreidimensionale Ordnung zurückzuschlüpfen. Ich will nicht erfahren, daß es den Dingen, wenn sie unbeobachtet sind, am Ende möglich wäre, aus der Disziplin der Naturgesetzte zu springen.«34)

Ein ähnlicher Text stammt von Ernst Bloch. Er trägt den Titel »Der Rücken der Dinge« und ist 1930 in der Sammlung Spuren erschienen: "[W]as die Möbel während unseres Ausgangs taten, ist dunkel. Keine Vermutung darüber ist zu beweisen, aber auch keine, noch so phantastische, zu widerlegen.«35) Vom Zimmer würden wir nur die vordere Seite kennen, aber kein Mensch wisse "noch, woraus der Rücken der Dinge besteht, [...] gar ihre Unterseite, und worin das Ganze schwimmt.« Man kenne nur die »Vorderseite oder Oberseite ihrer technischen Dienstwilligkeit«.36) Nach Jörg Zirfas lassen sich diese Gedanken einer frühen animistischen Phase der Kindheit zurechnen, für die man auch in Walter Benjamins Berliner Kindheit um 1900 eindrückliche Beispiele findet, dass nicht nur die Menschen oder Tiere, sondern auch Dinge leben. »In dieser Phase begreifen Kleinkinder nicht nur andere Menschen als sich ähnlich, sondern auch Gegenständen werden (projektiv) innere Vorgänge zugeschrieben [...].«37)

Diese Deutung, die sich auf die kindliche Wahrnehmung bezieht, und

34) Polgar, Alfred: Die Dinge [1926]. In: ders.: Kleine Schriften, Bd. 2. Ed. Reich-Ranicki, Marcel. Hamburg 1983, 17-21, hier 17-18. Vgl. Kimmich 2015 (wie Anm. 8), 187-188.

35) Bloch, Ernst: Spuren [1930]. Gesamtausgabe der Werke, Bd. 1. Frankfurt am Main: Suhrkamp Verl., 1969. 172-175, hier 172

36) Bloch 1969 (wie Anm. 35), 174-175.

37) Zirfas, Jörg: Die Performativität der Dinge. Pädagogische Reflexionen über Bildung und Design. In: Zeitschrift für Erziehungswissenschaft 16 (October 2013), 43-57, hier 44 . damit gleichsam eine anthropologische Konstante konstruiert, verpasst den durch und durch modernen Zugang Blochs zur Dingproblematik. Für Blochs kleine Skizze »Der Rücken der Dinge« drängt sich der Begriff „Dingtext « 38 ) (Dorothee Kimmich) oder »Dingprosa«39) (Uwe C. Steiner) auf. Als »Dingtexte« subsumiert Kimmich Texte der literarischen Moderne über Dinge, die »undeutbar und lebendig« sind.40) Steiners Begriff der »Dingprosak ist der Literatur über Dinge vorbehalten, in der sie als »autonom-agile, widerständige, fremde« erscheinen.41) Alle solchen Dinge stehen in der Vischerschen Tradition der »Tücke des Objekts«.42)

Solche Erfahrungen thematisieren moderne Texte von Franz Kafka, Siegfried Kracauer, Walter Benjamin, Robert Walser, Rainer Maria Rilke und Robert Musil in »besonders expliziter Weise«. Zugleich diskutieren sie auch Fragen, »ob es Möglichkeiten gibt, die Bedrohung in ,Solidarität", ,Freundschaft ${ }^{\star}$ oder am ehesten ,Komplizenschaft mit den Dingen umzuwandeln. «43)

In Robert Walsers Der Gehülfe (1908) steht eine Szene für eben diese Tücken der Objekte, worin er ganz beiläufig »die typischen Strukturelemente des Topos« aktualisiere, um sie schließlich »desinteressiert zu quittieren «,44) Walser schreibt: „Die Haare schienen heute früh außerordentlich schwer zu bürsten und zu kämmen zu sein. Die Zahnbürste erinnerte an vergangene

38) Kimmich 2011 (wie Anm. 11), 34

39) Steiner, Uwe C.: Dingprosa. Gegenstände als Subjekte des Alltags heute und in der kritischen Theorie. In: Preußer, Heinz-Peter; Visser, Anthomya (eds.): Alltag als Genre. Heidelberg: Winter, 2009, 13-31, hier 23.

40) Kimmich 2011 (wie Anm. 11), 33

41) Steiner 2009 (wie Anm. 39), 22.

42) Vgl. Steiner 2018 (wie Anm. 30), 249-256.

43) Kimmich 2018 (wie Anm. 7), 26.

44) Maas, Julia: Dinge, Sachen, Gegenstände. Spuren der materiellen Kultur im Werk Robert Walsers. München: Wilhelm Fink, 2019 (Robert Walser Studien; 2), 20. 
Zeiten. Die Seife, womit man die Hände waschen sollte, glitt aus, fuhr unters Bett, und man musste sich bücken und sie aus dem hintersten Winkel hervorziehen. Der Kragen war zu hoch und zu eng, obgleich er doch gestern prächtig gepasst hatte. Welche wunderbaren Dinge! Und wie langweilig das alles war.«45) Darauf folgt eine Analepse; Joseph Marti, der "Gehülfe« erinnert sich ähnlicher Geschichten mit Gebrauchsgegenständen. Er bezeichnet sie als "Kragengeschichten«, "Mäntelgeschichten« und "Schuhgeschichten«, wobei die Formulierung es offenlässt, ob Marti damit »seine« Geschichte mit diesen Objekten oder die Geschichte des jeweiligen Objekts meint: „Joseph erinnerte sich gewisser Zeiten in seinem Leben, wo ihn der Ankauf einer neuen Krawatte oder eines steifen englischen Hutes in seelische Aufregung versetzen konnte. Vor einem halben Jahr hatte er eine solche Hutgeschichte erlebt. Es war ein halbhoher, ganz guter, normaler Hut, wie ihn die ,bessern' Herren zu tragen pflegen. Er aber traute dem Hut nichts Gutes zu. Er setzte sich ihn tausendmal auf den Kopf, vor dem Spiegel, um ihn dann endlich auf den Tisch zu legen. Dann ging er drei Schritte weg von dem niedlichen Ungetüm und beobachtete ihn, wie ein Vorposten den Feind beobachtet. Es war nichts an ihm auszusetzen. Hierauf hängte er ihn an den Nagel, auch da erschien er ganz harmlos. Er versuchte es wieder mit dem Kopf, entsetzlich! Es schien ihn von unten bis oben zerspalten zu wollen. Er hatte das Gefuihl, als ob seine Persönlichkeit eine benebelte, gesalzene, halbierte geworden sei. Er trat auf die Straße: er schwankte wie ein schnöder Betrunkener, er fühlte sich wie verloren. Er trat in eine Erfrischungshalle, legte den Hut ab: gerettet! - Ja, das war eine Hutgeschichte gewesen.«(46) Die Geschichte führt noch einmal paradigmatisch die Tücke des Objekts vor.

45) Walser, Robert: Der Gehülfe [1908]. Roman, ed. Carl Seelig. Zürich: Buchclub Ex Libris, 1955,150

46) Walser 1955, 150-151 (wie Anm. 45). Vgl. Maas 2019 (wie Anm. 44), 21.
Ein Gegenstand des Gebrauchs lässt sich nicht reibungslos gebrauchen. Und es entsteht beim Helden der Verdacht, die Versagung geschehe mit Absicht. "Das verflixte Ding macht sich lustig über den Zweck, den wir ihm zugedacht haben, und gewinnt zugleich, wenn es uns Streiche spielt, einen Augenblick die Würde des Zwecklosen: es spielt mit uns.«47) Und wir müssen, so Adolf Muschg, die alltägliche Erfahrung machen, ,[d]ass wir am Ende von den Geschöpfen abhängen, die wir machen.«48) Der ganze Roman ist von Dingen, genauer von den wenigen »Siebensachen«, gerahmt, die Joseph Marti in seinem Gepäck mit sich trägt. Mit einem »braunen Koffer, einen von den ganz billigen« steht er vor dem Büro von Carl Tobler, der ihn für sechs Monate beschäftigen wird. Hierin enthalten sind $»$ die paar Wäschestücke, die er besaß«, sowie ein »Knäuel alter Fäden, Bindfadenteile, Halsbinden, Knöpfe, Nadeln und abgerissene Leinenfetzen«,49) Und am Ende macht Joseph sich daran, »seine paar Sachen einzupacken«, und er nimmt »der Reihe nach wieder diese kleinen nichts- und für ihn vielbedeutenden Gegenstände in die Hand, um sie säuberlich, aber rasch, in den bereitgehaltenen Koffer zu stecken. «50) Nach Maas sind Walsers Koffer mit Marginalien ausgestattet; sie beinhalten Gegenstände, deren Nutzen nicht immer unmittelbar ersichtlich sei, »mitunter solche, die beschädigt oder nur noch bruchstückhaft vorhanden sind.《(51)

Dass »die Wahl der mitgenommenen Dinge« vor allem der "Selbstrepräsentation des Subjekts in der Fremde« dient, hat Gisela Ecker in ihrer Studie zum Koffer in der Literatur herausgearbeitet. Das im Koffer eingeschlossene $»$ Portrait des Besitzers « 12 ) bleibe aber meist wegen seines

47) Muschg 1980 (wie Anm. 3), 5-6.

48) Muschg 1980 (wie Anm. 3), 8.

49) Walser 1955 (wie Anm. 45), 14.

50) Walser 1955 (wie Anm. 45), 311.

51) Maas 2019 (wie Anm. 44), 102. 
eindeutigen Gebrauchswertes unbemerkt. ${ }^{53)}$ Bei Walser ist es der Inhalt des Koffers, der gegen das literarische Gebot der Brauchbarkeit verstößt und ihn auffällig macht

Noch zwei Stellen seien aus dem Roman zitiert. Nach einem Zwischenfall mit Frau Tobler zitterte der "Gehülfe« heftig. "Es war ihm eine Unmöglichkeit, die Feder in der Hand zu halten. Das Leben selber tanzte ihm vor den Augen. Fenster, Tisch und Stühle schienen lebendige Wesen geworden zu sein. Er setzte den Hut auf und ging baden. (154) Und an einer späteren Stelle wird berichtet, Frau Tobler wischte ein wenig den Staub von den Möbeln; »aber sie rührte die Möbel eigentlich mehr nur so an, als würde sie dieselben haben liebkosen und grüßen wollen.(155) Im einen Falle ist das Leben der Dinge eine Frage der Wahrnehmung Martis, im anderen ein literarischer Vergleich.

Walsers Dinge, die er als Gegenstände paraphrasiere und durch Attribute hervorhebe, seien "scheinbar uninteressant, handlich und nützlich.«(56) Nach Robert Musil stellt Walser die Selbstverständlichkeit des gewohnten Dingbezugs in Frage: $» W i r$ haben in vielen Dingen so feste Verhaltensweisen unseres Gefühls, daß wir sie wie in den Dingen selbst gelegen behandeln«, die Lektüre von Walsertexten könne dem Abhilfe verschaffen und daher vergleicht Musil sie mit einem sjener scheinbar unnützen, trägen Tage, wo sich unsere festen Überzeugungen in eine angenehme Gleichgültigkeit lockern.«57) Walser sei ein "Dingschriftsteller« und die Dinge würden bei

52) Ecker, Gisela: Geschichten von Koffern. In: Bracher, Philip; Hertweck, Florian; Schröder, Stefan (eds.): Materialität auf Reisen. Zur kulturellen Transformation der Dinge. Berlin: LIT, 2006 (Reiseliteratur und Kulturanthropologie; 8), $215-$
232, hier 221-222.

53) Ecker 2006 (wie An. 52), 217

54) Walser 1955 (wie Anm, 45), 176-177.

55) Walser 1955 (wie Anm. 45), 243.

56) Maas 2019 (wie Anm. 44), 20. ihm einen »maßgeblichen Bestandteil seiner Poetologie« ausmachen, schreibt Julia Maas. 58 )

Dialogizität zwischen Mensch und Dingen: Béla Balázs (1884 - 1949), dem Theoretiker des (Stumm-)Films verdanken wir eine Untersuchung, Der sichtbare Mensch oder die Kultur des Films (1924), in der er grundlegend über den Menschen und die ihn umgebenden Gegenstände im Film nachdachte. Um seine Aussagen $\mathrm{zl}$ unterstiutzen, bringt er ein Zitat aus einer Textstelle von Goethe (er zitiert nach der Sophienausgabe), die dieser für die Physiognomischen Fragmente von Johann Caspar Lavater geschrieben hatte.59) Goethe schrieb: »Aber was ist das Äußere am Menschen? Wahrlich nicht seine nackte Gestalt, unbedachte Gebärden, die seine innern Kräfte und deren Spiel bezeichnen! Stand, Gewohnheit, Besitztümer, Kleider, alles modifiziert, alles verhüllt ihn. Durch alle diese Hüllen bis auf sein Innerstes zu dringen, selbst in diesen fremden Bestimmungen feste Punkte zu finden, von denen sich auf sein Wesen sicher schließen läßt, scheint äußerst schwer, ja unmöglich zu sein. Nur getrost! Was den Menschen umgibt, wirkt nicht allein auf ihn, er wirkt auch wieder zurück auf selbiges, und indem er sich modifizieren läßt, modifiziert er wieder rings um sich her. So lassen Kleider und Hausrat eines Mannes sicher auf dessen Charakter schließen. Die Natur bildet den Menschen, er bildet sich um, und diese Umbildung ist doch wieder natürlich; er, der sich in die große weite Welt gesetzt sieht, umzäunt, ummauert sich eine kleine drein, und staffiert sie aus nach seinem Bilde. $\langle(00)$

57) Musil, Robert: Die Geschichten von Robert Walser [1914]. In: Kerr, Katharina (ed.): Über Robert Walser, Bd.1. Frankfurt am Main: Suhrkamp Verl., 1978, 8991, hier 90 .

58) Maas 2019 (wie Anm. 44), 217.

59) Balázs, Béla: Schriften zum Film. Eds. Diederichs, Helmut H.; Gersch, Wolfgang; Nagy, Magda Bd. 1: „Der sichtbare Mensch [1924], Kritiken und Aufsätze 1922-1926. München: Carl Hanser Verl., 1982, 73. 
In dieser gegenseitig Modifizierung und Einwirkung findet Balázs seinen Grundgedanken. Im Stummfilm seien die Dinge, nicht wie in »der Welt des sprechenden Menschen [...] zurückversetzt und degradiert«, sondern sie würden "[i]n der gemeinsamen Stummheit [...] mit den Menschen fast homogen und gewinnen dadurch an Lebendigkeit und Bedeutung. « Und: "Weil sie nicht weniger sprechen als die Menschen, darum sagen sie gerade so viel.«(11) Beim Film würden wir ausschließlich nach dem Äußern urteilen, und da uns die Sprache keinerlei Aufschluss gebe, müsse ıjeder Charakter seine Symbole an sich tragen«, sonst würden wir die Bedeutung seines Handelns nicht verstehen. $\gg$ Denn dieselbe Tat kann gut und böse gemeint sein. Wir müssen es eben dem Menschen ansehen, wie er sie meint.«(62) Für Balázs haben Dinge ein Gesicht: »Jedes Kind kennt die Gesichter der Dinge und geht mit klopfendem Herzen durch das halbdunkle Zimmer, in dem Tisch und Schrank und Sofa wilde Grimassen schneiden und mit wunderlichem Mienenspiel etwa sagen wollen.«63) Die Dinge können einen aber auch freundlich anblicken. Es ist die »lebendige Physiognomik«, die allen Dingen eignet. Und das Kind kenne diese Physiognomien gut, nweil es die Dinge noch nicht ausschließlich als Gebrauchsgegenstände, Werkzeuge,

60) Die Stelle findet zum ersten Mal in Lavater, Johann Caspar: Physiognomische Fragmente, zur Beförderung der Menschenkenntni $\beta$ und Menschenliebe. Bd. 1. Leipzig/Winterthur: Weidmanns Erben und Reich; Heinrich Steiner, 1775, 15. Die genaue Zuschreibung der Beiträge Goethes zu den Physiognomischen Fragmenten gelang zuerst Eduard von der Hellen; vgl. von der Hellen, Eduard: Goethes Anteil an Lavaters physiognomischen Fragmenten. Frankfurt am Main: Rütten, 1888. Im vorliegenden Aufsatz wurde zitiert nach Goethe, Johann Wolfgang: Der junge Goethe 1757-1775. Ed. Sauder, Gerhard. Sämtliche Werke nach Epochen seines Schaffens | Münchner Ausgabe, Bd. 1.2. München: Carl Hanser Verl., 1987, 458-
461, 863-867, hier 458.

61) Balázs 1982 (wie Anm. 59), 66 (Hervorhebung im Original).

62) Balázs 1982 (wie Anm. 59), 72.

63) Balázs 1982 (wie Anm. 59), 92.
Mittel zum Zweck ansieht [...]. Es sieht in jedem Ding ein autonomes Lebewesen, das eine eigene Seele und ein eigenes Gesicht hat.(164) Wichtig für Balázs ist, in Anlehnung an Goethe, dass das den Körper des Filmschauspielers umgebende Milieu (die Welt der Dinge) als nsichtbare „Aura'« zu seiner »über die Konturen des Körpers erweiterten Physiognomie« wird.65) Der Filmschauspieler ist über die ihn umgebenden Dinge fassbar und sein Spiel deutet das Mienenspiel und Gebärdenspiel der Dinge. Für den Marxisten Balázs soll auch die Fabrikmaschine ein Gesicht bekommen - die Fratze eines Molchs.66) Und schließlich: Warum man literarische Gleichnisse nicht in Filmbilder »übersetzen« soll, hat damit $z u$ tun, dass Bilder »immer eine Gegenwart« haben: "Das heißt, die Dinge haben ihre eigene Wirklichkeit und außerdem noch eine weitere Bedeutung. Aber nur jene Bedeutung zu haben, ohne unmittelbare eigene Realität, das macht das Bild jedes Dinges zu einer toten und leeren Vignette. $\left\langle\left\langle^{67)}\right.\right.$ Allerdings werden die »Mienen der Dinge« - und hier zieht Balázs wieder die alte Hierarchie von Subjekt (Mensch) und Objekt (Dinge, Sache, Gegenstand) ein - nur insoferm bedeutsam, »als sie eine Beziehung zum Menschen haben. $\left({ }^{68)}\right.$

Die Zunehmende Beherrschung der Welt bzw. der Dingwelt, die durch die industrielle Produktion ab 1800 auch quantitativ zunimmt (wir besitzen immer mehr Gegenstände, Dinge, Sachen, Zeug, Objekte) macht sich nun dadurch bemerkbar, dass es die Dinge sind, die uns beherrschen. Auf diese Dialektik, die intellektuell erst um 1900 reflektiert wurde, reagiert die Literatur schon früh: In populären Märchen, wie sie die Grimms erzählten und herausgaben, in Kunstmärchen eines Hans Christian Andersen, im realistischen Roman

64) Balázs 1982 (wie Anm. 59), 92

65) Balázs 1982 (wie Anm. 59), 98.

66) Vgl. Balázs 1982 (wie Anm. 59), 101-103.

67) Balázs 1982 (wie Anm. 59), 104-105.

68) Balázs 1982 (wie Anm. 59), 98. 
eines Friedrich Theodor Vischers treten Objekte als aktive, die Handlung beherrschende oder diese erleidende Subjekte auf. Sie können sprechen (direkte Rede), Beziehungen mit anderen Objekten eingehen, sie weisen Lebensläufe auf (zwischen Geburt und Tod), mitunter sind sie (fast) unsterblich (Kafkas Odradek). Das Moderne an ihnen ist der Umstand, dass sie bedrohliche, unheimliche Züge annehmen können, die im Grunde unser schlechtes Gewissen reflektieren. Schriftsteller phantasieren deshalb den "Aufstand der Dinge« (Erhart Kästner) und es werden literarische Strategien erprobt, die Dinge mit uns wieder zu versöhnen.

Gerade das "Sammeln« von Dingen - in Läden, auf der Straße, in Texten - ist ein zentrales Motiv bei Benjamin. Sammler und Sammlungen, das Sammeln und Ordnen von Dingen, Büchern, Glasscherben, Spulen, Spielzeug etc. ist bei Benjamin ein vielfach variiertes Motiv. Jede Sammlung, so Benjamin, folge einer bestimmten Logik, habe eigene Gesetze. Sammeln zielt auf Vollständigkeit der Sammlung, aber zugleich fürchtet der Sammler natürlich auch nichts mehr als das Ende seiner Sammelmöglichkeiten und damit das Ende aller seiner Sehnsüchte. Sammeln ist auf diese Weise eingespannt zwischen sehr physischen (weitersammeln) und sehr metaphysischen (Vollständigkeit) Begierden und Wünsche.

Offenbar enthält das Verhältnis des Sammlers zu den Dingen, »das in ihnen nicht ihren Funktionswert, also ihren Nutzen, ihre Brauchbarkeit in den Vordergrund rückt, sondern sie als den Schauplatz, das Theater ihres Schicksals studiert und liebt«,69) das Geheimnis einer ganz besonderen, fast zauberhaften Beziehung zu den Dingen. Für den Sammler gleicht jedes Ding einer »magischen Enzyklopädie«. "Sammler sind Physiognomiker der

69) Benjamin, Walter: Ich packe meine

[1931]. In: ders.: Gesammelte Schrifien

Verl., 1981, 388-396, 997-998, hier 389.
Dingwelt«. ${ }^{70)}$ Sie schauen sich die Oberfläche, die Außenseite, die Gesichter der Dinge genau an. Sie sind die Spezialisten für die Stofflichkeit, für Form, Farbe, Geruch und Textur der Dinge, für deren Materialität, nicht für ihre Brauchbarkeit.

70) Benjamin, Walter: Lob der Puppe. Kritische Glossen zu Max v. Boehns »Puppen und Puppenspiele« [1930]. In: ders.: Gesammelte Schriften, Bd. III, ed. Tiedemann-Bartels, Hella. Frankfurt am Main: Suhrkamp Verl., (1972) ${ }^{3} 1989,213-218$, 638, hier 217. 


\section{Literaturverzeichnis}

\section{Quellen}

Andersen, H.[ans] C.[hristian]: Der Halskragen [1847]. In: ders.: Ausgewählte Werke, Bd. 5: Gesammelte Märchen. Leipzig: Verl. Carl B. Lorck, 1849.

Balázs, Béla: Schriften zum Film. Eds. Diederichs, Helmut H.; Gersch, Wolfgang; Nagy, Magda. Bd. 1: ,Der sichtbare Mensch', Kritiken und Aufsätze 1922 - 1926. München: Carl Hanser Verl., 1982.

Benjamin, Walter: Lob der Puppe. Kritische Glossen zu Max v. Boehns »Puppen und Puppenspiele« [1930]. In: ders.: Gesammelte Schriften, Bd. III, ed. Tiedemann-Bartels, Hella. Frankfurt am Main: Suhrkamp Verl., (1972) ${ }^{3} 1989,213-218,638$.

Benjamin, Walter: Ich packe meine Bibliothek aus. Eine Rede über das Sammeln [1931]. In: ders.: Gesammelte Schriften, Bd. IV, ed. Rexroth, Tillman. Frankfurt am Main: Suhrkamp Verl., 1981, 388 -
396, 997 - 998.

Blackwell, Mark; Bellamy, Liz; Keenleyside, Heather; Lupton, Christina (eds.): British it-narratives, 1750 - 1830, 4 Bde. London: Pickering \& Chatto, 2012.

Bloch, Ernst: Spuren [1930]. Gesamtausgabe der Werke, Bd. 1. Frankfurt am Main: Suhrkamp Verl., 1969. 172 - 175.

Böll, Heinrich: Schicksal einer henkellosen Tasse. In: ders.: Gesammelte Erzählungen, 2 Bde. Köln: Kiepenheuer \& Witsch, 1981, 368 - 374.

Engels, Friedrich; Marx, Karl: Die heilige Familie, oder Kritik der kritischen Kritik. Gegen Bruno Bauer \& Consorten. Frankfurt am Main: Literarische Anstalt, 1845.

Gabricesvkij, Aleksander: Die Sprache der Dinge. In: Hennig, Anke (ed.): Uber die Dinge. Texte der russischen Avantgarde. Hamburg: Philo Fine Arts, 2010 (Findus-Bücher; 181), 567 - 579.
Grimm, [Jacob und Wilhelm]: Kinder und Hausmärchen. Gesammelt durch die Brüder G. Große Ausgabe. 2. Bde. Göttingen: Verlag der Dieterichschen Buchhandlung, (1812/1815) ${ }^{7} 1857$.

Goethe, Johanna Wolfgang: Der junge Goethe 1757-1775. Ed. Sauder, Gerhard. Sämtliche Werke nach Epochen seines Schaffens | Münchner Ausgabe, Bd. 1.2. München: Carl Hanser Verl., 1987.

Krohn, Tim: Aus dem Leben einer Matratze bester Machart. Zürich: Diogenes Verl., (2014) 2015.

Lavater, Johann Caspar: Physiognomische Fragmente, zur Beförderung der Menschenkenntniß und Menschenliebe. Bd. 1. Leipzig/ Winterthur: Weidmanns Erben und Reich; Heinrich Steiner, 1775.

Muschg, Adolf: Die Tücke des verbesserten Objekts. Wald: Verl. Im Waldgut, 1980 (Die Reihe im Waldgut; 3).

Musil, Robert: Die Geschichten von Robert Walser [1914]. In: Kerr, Katharina (ed.): Über Robert Walser, Bd.1. Frankfurt am Main: Suhrkamp Verl., 1978, 89 - 91.

Polgar, Alfred: Die Dinge [1926]. In: ders.; Kleine Schriften, Bd. 2: Kreislauf. Ed. Reich-Ranicki, Marcel. Reinbek bei Hamburg: Rowohlt, 1983, $17-21$.

Vischer, Friedrich Theodor. Ed. Ulrich Ott. Marbach: Deutsche Schillergesellschaft Marbach am Neckar, 1987 (= Marbacher Magazin, 44).

Walser, Robert: Der Gehülfe [1908]. Roman, ed. Carl Seelig. Zürich: Buchclub Ex Libris, 1955.

\section{Sekundärliteratur}

Böhme, Hartmut: Fetischismus und Kultur. Eine andere Theorie der Modeme. Reinbek bei Hamburg: Rowohlt Taschenbuch Verl., (2006) ${ }^{2} 2006$ (Rowohlts Enzyklopädie; 55677).

Brunner, José (ed.): Erzählte Dinge. Mensch-Objekt-Beziehungen in der 
deutschen Literatur. Göttingen: Wallstein, 2015 (Schriftenreihe des Minerva Instituts für deutsche Geschichte der Universität Teihe des 32).

Ecker, Gisela: Geschichten von Koffern. In: Bracher, Philip; Hertweck, Florian; Schröder, Stefan (eds.): Materialität auf Reisen. Zur kulturellen Transformation der Dinge. Berlin: LIT, 2006 (Reiseliteratur und Kulturanthropologie; 8), $215-232$.

Hahn, Hans Peter: Materielle Kultur. Eine Einführung. Berlin: Reimer, 2005.

Hahn, Hans Peter: Vom Eigensinn der Dinge. In: Bayerisches Jahrbuch fü Volkskunde (2013), $13-22$.

Kimmich, Dorothee: Lebendige Dinge bei Walter Benjamin und Robert Walser. In: Dogilmunhak. Koreanische Zeitschrift für Germanistik 110 (2009), 9 - 29.

Kimmich, Dorothee: Lebendige Dinge in der Moderne. Konstanz: Konstanz University Press, 2011.

Kimmich, Dorothee: Der Fremde und seine Dinge. Bemerkungen zur Funktion fremder Dinge in der Literatur der Moderne. In: Brunner 2015, 177 -
188.

Kimmich, Dorothee: Dinge in Texten. In: Scholz/Vedder 2018, 21 - 28.

Knipp, Raphaela: Narrative der Dinge. Literarische Modellierungen von Mensch-Ding-Beziehungen. In: Zeitschrift für Literaturwissenschaft und Linguistik 42. Jg., H. 168 (2012), 46-61.

Körte, Mona: Der Un-Sinn der Dinge in Märchentexten um 1800. In Zeitschrift für Germanistik, NF 22 (2012), 57 - 71.

Körte, Mona: Dinge im Diminutiv. Der Eigensinn des Kleinen in der "Kinder- und Hausmärchen« der Brüder Grimm. In: Brunner 2015, 21 36.

Körte, Mona: Volks- und Kunstmärchen. In: Scholz/Vedder 2018, 214 - 221.

Löffler, Klara: Wenn Buster Keaton und Erwin Wurm Häuser verrücken. Ein explorativer Vergleich. In: Dittmeyer, Daria; Hommers, Jeannet;
Windmüller, Sonja (eds.): Verrückt, Verrutscht, Versetzt. Zur Verschiebung von Gegenständen, Körpern und Orten. Berlin: Reimer Verl., 2015 (Schriftenreihe der Isa Lohmann-Siemens Stiftung; 8), 46 -54 .

Maas, Julia: Dinge, Sachen, Gegenstände. Spuren der materiellen Kultur im Werk Robert Walsers. München: Wilhelm Fink, 2019 (Robert Walser Studien; 2).

Müller-Wille, Klaus: Collagen, Wortdinge und stumme Bücher. Hans Christian Andersens (inter)materielle Poetik: Strässle, Thomas; Kleinschmidt, Christoph; Mohs, Johanne (eds.): Das Zusammenspiel der Materialien in den Künsten. Theorien - Praktiken - Perspektiven. Bielefeld: transcript Verl., 2013, 183 - 219.

Niehaus, Michael: Das Buch der wandernden Dinge. Vom Ring des Polykrates bis zum entwendeten Brief. München: Carl Hanser Verl., 2009.

Niehaus, Michael: Dinge in Bewegung. In: Samida, Stefanie; Eggert, Manfred K. H.; Hahn, Hans Peter (eds.): Handbuch Materielle Kultur. Bedeutungen, Konzepte, Disziplinen. Stuttgart/Weimar: Metzler Verl., 2014, 132 - 140.

Niehaus, Michael: Objektbiografien. In: In: Scholz/Vedder 2018, 239 - 247.

Scholz, Susanne; Vedder, Ulrike (eds.): Handbuch Literatur und Materielle Kultur. Berlin/Boston: De Gruyter, 2018 (Handbücher zur kulturwissenschaftlichen Philologie; 6).

Steiner, Uwe C.: Dingprosa. Gegenstände als Subjekte des Alltags heute und in der kritischen Theorie. In: Preußer, Heinz-Peter; Visser, Anthomya (eds.): Alltag als Genre. Heidelberg: Winter, 2009, 13 - 31.

Steiner, Uwe C.: Die Verrückung der anthropologischen Matrix. Vom Verhalten der Dinge bei Franz Kafka. In: Brunner 2015, 159 - 176.

Steiner, Uwe C.: Die Tücke des Objekts. Friedrich Theodor Vischers Auch Einer (1878). In: ScholzVedder 2018, 249-256. 
Vedder, Urike: Sprache der Dinge. In: Scholz/Vedder 2018, 29 - 37

von der Hellen, Eduard: Goethes Anteil an Lavaters physiognomischen Fragmenten. Frankfurt am Main: Rütten, 1888.

Zirfas, Jörg: Die Performativität der Dinge. Pädagogische Reflexionen über Bildung und Design. In: Zeitschrift für Erziehungswissenschaft 16 (2013), 43 - 57.
Zusammenfassung

\section{Dinge als Aktanten in der Literatur}

\section{Alfred Messerli (Uni Zürich, Schweiz)}

Ausdruck der zunehmenden Beherrschung der Welt ist im Zuge der industriellen Revolution ab 1800 die industrielle Produktion; seitdem besitzen die Subjekte zunehmend mehr Gegenstände, Dinge, Sachen, Zeug, Objekte. Diese Zunahme macht sich unter anderem dadurch bemerkbar, dass es nun die Dinge sind, die uns beherrschen. Auf diese Dialektik, die intellektuell erst um 1900 reflektiert wird, reagiert die Literatur schon früh: In populären Märchen, wie sie Jacob und Wilhelm Grimms erzählten und herausgaben, in Kunstmärchen eines Hans Christian Andersen, im realistischen Roman eines Friedrich Theodor Vischers treten Objekte als aktive, die Handlung beherrschende oder diese erleidenden Subjekte auf. Sie können sprechen (direkte Rede), Beziehungen mit anderen Objekten eingehen, sie weisen Lebensläufe auf (Dingbiografien), mitunter sind sie (fast) unsterblich (so Kafkas Odradek). Das Moderne an ihnen ist der Umstand, dass sie bedrohliche, unheimliche Züge annehmen können, die im Grunde unser schlechtes Gewissen reflektieren. Schriftsteller phantasieren deshalb den "Aufstand der Dinge« (Erhart Kästner) und es werden literarische Strategien erprobt, die Dinge mit uns wieder zu versöhnen. Zum Verständnis dieser Prozesse und Textstrategien, die mit dem material turn ins Blickfeld geraten, spielt die Literaturwissenschaft eine besondere Rolle. Sie ist das wichtige Medium, in welchem die Probleme mit den Dingen, ihr Eigensinn, ihre unbekannte Seite, ihre »Ambivalenz von Zeichenhaftigkeit und zugleich nicht Zeichenhaftigkeit» (Dorothee Kimmich), ihre Opazität, ihre Geschichte reflektiert und auserzählt werden kann. 
Schlüsselbegriffe: Ding als Aktant, material turn, Dingbiografie, Tücke des Objekts, Zusammenleben mit Dingen
국문요약

\section{문학에 나타난 행위자로서의 사물}

알프레드 메설리 (스위스 취리히 대학)

산업혁명이 시작된 1800 년경 이래로 점점 강력해지는 인간의 세계 지배는 공산품의 생산이라는 형태로 표출되었다. 이 때부터 주체들은 점점 더 많은 대상, 사물, 물건, 객체를 소유하게 되었다. 이러한 사물의 증가는 무엇보다도 이제 역으로 사물들이 우리를 지배하는 현상에서 두드러지게 나타난다. 이러 한 변증법은 1900 년경에 들어서야 지성적으로 성찰되기 시작하였으나, 문학 은 이미 일찍부터 이에 반응하고 있었다. 예컨대 야콥 그림과 빌헬름 그림이 서술하고 펴낸 것과 같은 민중 메르헨, 한스 크리스티안 안데르센 풍의 창작 메르헨, 그리고 프리드리히 테오도르 피셔 풍의 사실주의적 소설에서 사물들 은 능동적으로 행동을 지배하거나 이것을 격는 주체들로 등장한다. 그것들은 말을 할 수 있고 (직접 화법), 다른 사물들과 관계를 맺을 수 있으며, 삶의 이 력들을 보여 주고 (사물의 전기), 때로는 (거의) 불사의 존재이기도 하다 (카 프카의 오드라덱처럼). 이 사물들이 위협적이고 섬뜩한 성격을 띨 수 있다는 점은 현대적인데, 이는 사실상 우리가 느끼는 양심의 가책을 반영하는 것이 다. 그렇기 때문에 작가들은 “사물들의 반란'(에르하르트 케스트너)을 상상하 기도 하며, 사물들과 우리를 다시 화해시키기 위한 문학적 전략들을 시험해 본다. '물질적 전회'와 더불어 주목받기 시작한 이러한 과정과 텍스트적 전략 을 이해하는 데에는 문학 연구가 중요한 역할을 한다. 문학 연구는 사물과 관 련된 제 문제, 사물들의 고집과 숨겨진 면모, “상징적이면서도 동시에 상징적 이지 않다는 양가성"(도로테 키미히), 불투명성과 역사를 성찰하고 충분히 서 술할 수 있게 해 주는 중요한 매체이다. 
주제어: 행위자로서의 사물, 물질적 전회, 사물의 전기, 객체의 술책, 사물들과 함께 살아가기

필자 E-Mail 주소: amesser@bluewin.ch

논문투고일: 2019. 11. 10, 논문심사일: 2019. 12. 11, 게재확정일: 2019. 12. 12. 